\title{
On the Spectral Coexistence of GSO and NGSO FSS Systems: Power Control Mechanisms and A Methodology for Inter-site Distance Determination
}

\author{
A. Pourmoghadas*, S. K. Sharma, S. Chatzinotas, B. Ottersten \\ Interdisciplinary Centre for Security, Reliability and Trust (SnT), University of Luxembourg L-2721, \\ Luxembourg.
}

\begin{abstract}
SUMMARY
The co-existence of geostationary satellite orbit (GSO) and non-geostationary satellite orbit (NGSO) fixed satellite service (FSS) systems within the same spectrum to enhance the spectrum efficiency in Ka-band has attracted a lot of interest lately. However, the co-existence of GSONGSO satellite systems with limited shared spectrum can cause in-line interference from a satellite to other satellite's earth terminals. In this context, this contribution investigates three possible power control approaches to mitigate the in-line interference caused by an NGSO satellite to the GSO earth terminal, while the NGSO satellite is crossing the GSO satellite's illumination zone. Moreover, three types of interference mitigation techniques, namely range-based (R-PC), trafficaware (TA-PC) and cognitive (C-PC) power control techniques are investigated. Furthermore, to increase the spectrum efficiency of the GSO-NGSO FSS terrestrial network, we formulate and solve an optimization problem with the objective of minimizing the inter-site distance (ISD) between earth user-terminals. More specifically, we find the minimum distance from a GSO earth terminal, where an NGSO earth terminal can be implemented subject to minimizing received interference level at the victim system. Finally, our comparative results show that the cognitive power control technique performs the best in protecting the victim receiver from the in-line interference. Copyright (c) 2016 John Wiley \& Sons, Ltd.
\end{abstract}

Received ...

KEY WORDS: Cognitive Power Control; Range-based Power Control; Traffic-Aware Power Control; Inter-site Distance; Interference Mitigation; GSO; NGSO

\section{INTRODUCTION}

The expected traffic volume increase in wireless communications, the need for ubiquitous coverage and high quality services with the limited available resources are putting strain on the existing infrastructure of communication systems across the globe. Satellite communications offers many possibilities and opportunities in achieving such efficiency in quality of service and extending backhaul services to anywhere and any-time. However, due to growing rate on the number of operating satellites, satellite systems are also facing the limitation on orbits and frequency bands. Studies on efficient usage of satellite resources and their co-existence with either terrestrial systems and other satellite networks exist in the literature [1]- [2]. The co-existence of satellite systems with either other satellite systems or terrestrial networks can be made possible by employing suitable resource allocation and interference mitigation techniques [3], [5]. The traditional resource allocation techniques are

\footnotetext{
${ }^{*}$ Correspondence to: Interdisciplinary Centre for Security, Reliability and Trust (SnT), University of Luxembourg, 4, rue Alphonse Weicker, L-2721, Luxembourg, Email: ameneh.pourmoghaddaslangroudi@uni.lu.
} 
no longer efficient since a significant amount of the licensed spectrum remains underutilized, as reported by Federal Communication Commission [4].

Apart from the requirement of finding intelligent (or cognitive) resource allocation, it is important to ensure that the co-existence of the multi-satellite systems does not cause interference to other co-channel systems such as fixed satellite services, mobile satellite services (MSS), or terrestrial network. For instance, consider the co-existence of many GSO satellite systems operating over Europe and North America at the $\mathrm{Ka} / \mathrm{ku}$-band, which are located $3^{\circ}$ apart from each other to avoid the potential interference from an adjacent satellite to the earth terminal in the downlink communication. However, the interference mitigation is more complicated considering a practical dual satellite system (DSS) scenario, where two satellites (e.g., GSO-NGSO satellites) operate simultaneously over a coverage area while sharing the spectrum bands [6].

In the co-existence scenarios of GSO-NGSO networks, "in-line" interference arises whenever an NGSO satellite passes through a line of sight path between an earth station and a GSO satellite [7]. It occurs due to the fact that an NGSO satellite may create/receive interference through its sidelobe or mainlobe to the GSO system (please see Fig. 1). To understand this scenario better, we can consider the co-existence of O3b satellites within $\pm 5^{\circ}$ latitude from the equator and GSO Eutelsat KA-SAT satellite [orbit: 9.0, incl: 0.04$]^{\dagger}$ as a good example. O3b satellite which uses the MEO constellation and shares the frequency 18.8-19.3 GHz in downlink communication with the GSO system, has a high potential to cause interference to the GSO FSS system. In this context, one of the key challenges that has been identified in ITU Radio Regulations and European Space Agency is the need to explore efficient techniques to mitigate the in-line interference for the spectral co-existence of GSO-NGSO satellite networks.

\subsection{Related Literature}

Studies on interference mitigation techniques have been conducted, and various schemes to reduce the interference from other radio systems have been recommended in ITU-R reports and in the literature [1]- [10]. In general, techniques that are commonly used to facilitate the spectrum sharing between GSO and NGSO satellites can be divided into two categories as follows.

1) Coordinated techniques: In this category of techniques, a coordinator takes care of the synchronization between systems and sends the operating orders to the satellite stations. Some of these techniques are briefly described in the following.

(i) Frequency allocation: This technique is associated with a command center located either on the earth or on-board, which coordinates the frequency allocation for GSONGSO satellite systems during the interference [9].

(ii) Frequency channelization (or band planning): In this scheme, each frequency band is divided into authorized small sub-bands. Each sub-band is assigned to a separate beam, i.e., spatially separated from its nearest co-frequency beam. This division can improve the interference level by decreasing the probability of overlap between frequency bands [10].

(iii) RF monitoring and interference detection: Another technique to mitigate against the disruptive effects of satellite interference is rapid detection and characterization through effective monitoring, which leads to extracting the interruption frequency band whenever available [12].

(iv) Satellite diversity: This scheme is in accordance with a handover process due to selection of another satellite to avoid the interference. This technique tries to avoid

\footnotetext{
${ }^{\dagger}$ Orbit position is the longitude position around the geostationary orbit. The inclination angle shows that the GSO satellite moves north-south across the equatorial plane on a daily basis (http://www.satellitecalculations.com/Satellite/Catalog/catalogID.php?37258).
} 
main beam to main beam interference by switching traffic to an alternative satellite in view whenever such in-line events occur [11].

2) Uncoordinated (cognitive) techniques: This category of techniques deals with techniques that do not require prior coordination in order to enable co-existence of FSS systems. Some of these approaches are:

(i) Satellite selection strategies: In general, earth stations communicate with satellite stations in the highest elevation angle. A different tracking technique, such as selecting the satellite that has the largest angular discrimination with respect to the satellites of other NGSO FSS systems, may improve the system capacity [13].

(ii) Spot turnoff method: In this method, one of the two spots is turned off whenever two spots overlap too much [14] or turning off the transmitters whenever the antenna axis of NGSO satellites moves within a given angular distance of the GSO [15].

(iii) Power control technique: In this scheme, a cognitive transmission power technique is used to provide the required signal to noise ratio (SNR) at the receiver and maintain the interference level on the victim receiver, in the co-existence of the GSO-NGSO networks [7].

\subsection{Motivation And Contributions}

Power control techniques have been utilized on the current operating satellites. For instance, Intelsat designs a transmission plan that optimizes the amount of power and bandwidth based on the antenna size and bandwidth of the wanted receiver on the earth [29]. However, cognitive transmission and techniques for interference mitigation in such multi/dual satellite communication systems have not been deployed yet, and need further investigation [3].

Although enormous number of literature have addressed dynamic power control techniques for terrestrial cellular and cognitive radio networks [16]- [20], the applications of these techniques in the GSO-NGSO coexistence scenario is relatively new. The earlier research on power adaptation belongs to 19th century. For instance, [17]- [18] mainly focus on adaptive transmission power for the base-stations (BSs) to compensate the channel fading and controlling interference.

Wherein, [19] initiated the trend of energy efficient power control for cellular networks by applying a dynamic power control in downlink transmission. Authors in [19], propose a tardy scheduling, which schedules packet transmissions as slowly as possible to minimize power consumption subject to maintain the packet delay constraints.

Most of the studies on dynamic transmission power controls on satellite stations involves on-board energy saving, regardless of investigations on controlling the imposed interferences on the victim receiver. For example, $[21,22]$ focus on energy saving of the batteries on the satellite boards as a single unit. Whereas, [23] considers the energy consumption reduction of eclipsed satellites constellations as a whole system. In [23], authors provide a heuristic algorithm for an intelligent LEO constellations satellites to save energy in whole LEO constellation system based on the traffic demands from the earth. Besides, most of the research works in the literature have been carried out on operating individual satellite systems. Wherein, the integration between different satellite constellations plays a key role in moving towards the next generation of the satellite networks. In this regard, [24] and [25] provide a study on the co-existence of hierarchical multi-layered satellite network. Authors in [26] studied the effect of the attenuation/fading on the received interference level of the victim earth terminal from an adjacent satellite. Besides, the contribution in [27] studied the quality of service for the wanted earth receiver with a dynamic power control on satellite in a dual satellite system. Main concerns in [27] are to combat the propagation challenges due to climate change, like rain attenuation by controlling the on-board satellite transmission power.

A more related article to the objective of current paper can be found in $[7,8,28]$. The authors 
in [28] find the minimum separation distance for frequency sharing between fixed service (FS) earth terminal and terrestrial base station (BS). In [28], the interference from satellite to the earth terminals is omitted, and the paper concentrates on the interference caused from the terrestrial BS to the FS earth terminal. Wherein, [8] studies a dynamic joint power and carrier allocation technique for the cognitive satellite communications with terrestrial networks. The dynamic power on the satellite system is in associated with the user rate demand to avoid the harmful interference on the terrestrial networks.

According to the ITU-R S.1325-3 recommendation, adaptive power control on range in the downlink is one of the useful interference mitigation techniques in facilitating spectrum sharing between GSO and NGSO networks. This technique is left for further investigation in ITU-R S.1325-3. Also, to the best of our knowledge, an investigation on the traffic-aware power control technique as an interference mitigation method for the GSO-NGSO systems has not been carried out. Besides, studies on earth terminal deployment strategies is one of the possible approaches for the efficient use of limited spectrum bands and network's capacity improvement [28].

In this context, the main contributions of this paper are as follows:

(i) We apply the range-based power control (R-PC) technique in the GSO-NGSO spectral coexistence scenario.

(ii) We utilize the cognitive power control in [7] based on the range ${ }^{\ddagger}(\mathrm{C}-\mathrm{PC})$ in the coexistence scenario of GSO and NGSO FSS systems.

(iii) We analyse the feasibility of the cognitive range-based power control method in terms of GSO-NGSO geometry.

(iv) We formulate and solve the traffic-aware power control (TA-PC) problem based on ITU-R S.1325-3.

(v) We develop a model for minimum possible inter-site distance between the GSO and NGSO FSS earth terminals through analysis.

The rest of the paper is as follows: Section 2 provides the system model and the in-line interference scenario. The range-based, cognitive and traffic-aware power control methods are defined in Section 3. In Section 4, we propose a method for determining the minimum distance between GSO and NGSO earth terminals by relocating the NGSO earth terminal. Section 5 provides the analysis and simulation results. Finally, we draw the conclusion in Section 6.

\section{SYSTEM MODEL AND INTERFERENCE SCENARIO}

Unlike the NGSO satellites, whose position varies by time with respect to a certain geographical point on the earth surface, the position of the GSO satellites remains fixed. Therefore, the propagation distance from the earth surface to the GSO satellite and vice versa can be considered constant. Whereas, the propagation distance between the satellite to the earth surface on the LEO or MEO orbit varies by time; for instance, O3b satellite orbit period on the MEO constellation is about 4 hours. Following the spectrum sharing in Kaband in the co-existence of GSO-NGSO networks, ITU's 1995 World Radiocommunication Conference (WRC-95) has allocated the frequency bands 18.8-19.3 GHz (in downlink from space to Earth) and 28.6-29.1 GHz (in uplink from Earth to space) to the GSO -NGSO FSS networks, which we will be using in this work. In accordance with the co-existence of GSO-NGSO FSS system while sharing spectrum on downlink ${ }^{\S}$ and uplink, the following in-line interference scenarios are probable to occur (please see Fig. 1).

\footnotetext{
‡Throughout the article, range is the distance between NGSO satellite and an NGSO earth terminal.

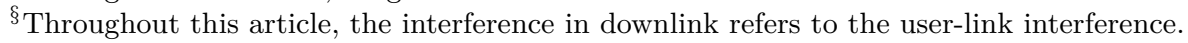




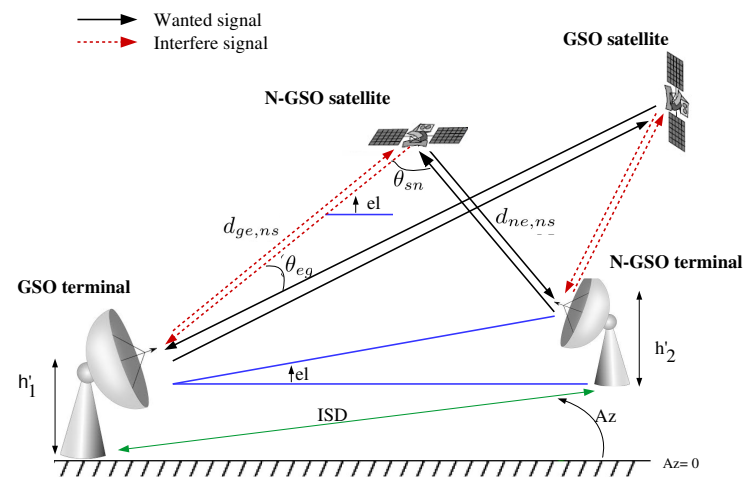

Figure 1. The interference scenario between GSO and NGSO FSS systems.

1. Interference from the NGSO satellite to the GSO earth terminal in the downlink.

2. Interference from the GSO earth terminal to the NGSO satellite in the uplink.

3. Interference from the GSO satellite to the NGSO earth terminal in the downlink.

4. Interference from the NGSO earth terminal to the GSO satellite in the uplink.

In this paper, we consider the GEO and MEO constellations, where O3b satellite in MEO orbit, and Eutelsat in GEO orbit are chosen as the use case scenario causing harmful in-line interference to the GSO earth terminal. Earth terminals are the user terminals operating in Ka-band in the FSS system such as VSAT. The results in ITU-R S.1325-3 indicate that the number and duration of interference events from NGSO system on GSO network in downlink is almost $90 \%$ of the caused interferences on the uplink feeder links. Therefore, in this work, we focus on the downlink in-line interference from NGSO satellite to the GSO earth terminal (item (1) from the above listed bullets, please see Fig. 1), and we leave the rest of the scenarios for our future work. Throughout this paper, we have assumed that the transmitter of the NGSO satellite communicates with their wanted receivers (NGSO earth terminal) along the boresight direction (i.e., off-axis angle $=0^{\circ}$ ); which means that the antenna gain of the NGSO satellite and the NGSO earth terminal remains fixed in the downlink communication. In our system model, we have assumed free space loss (FSL) on the direct or interference channels, and fading phenomena such as diffraction or tropospheric propagation effects etc., are not taken into account. We left this part for our future work.

\section{RANGE-BASED, COGNITIVE \& TRAFFIC-AWARE POWER CONTROL ALGORITHMS}

The power control method recommended by ITU-R S.1325-3 indicates that the NGSO satellite system can decrease or increase the transmission power (in downlink communication) based on the range, while providing the SNR level to the NGSO earth terminal. We call it as a range-based power control (R-PC) method. This method does not apply any further rules for protecting the GSO earth terminal from the created in-line interference. To enhance this method, we propose a cognitive range-based power control (CPC) algorithm at the NGSO satellite. In the proposed algorithm, the NGSO satellite will decrease or increase its transmission power while its distance to the NGSO earth terminal is getting shorter or longer accordingly. We have considered two threshold values for optimizing the transmission power of the NGSO satellite. The minimum SNR level at the wanted 


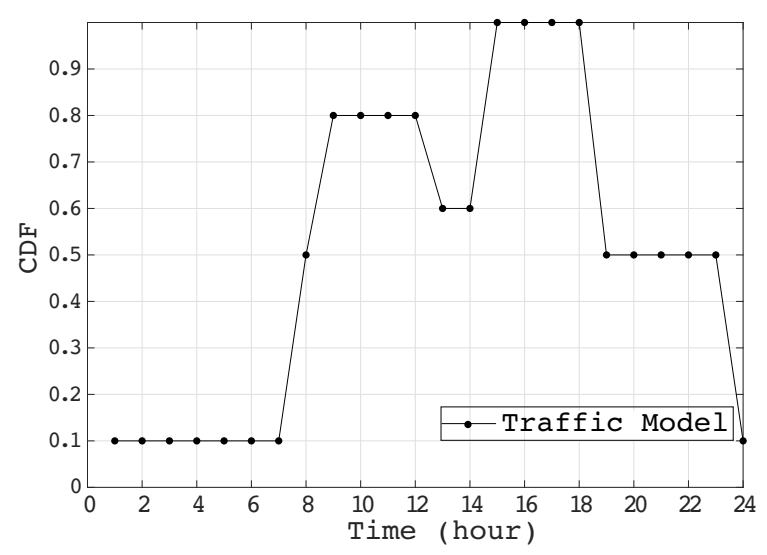

Figure 2. Traffic load variation at the NGSO earth terminal with respect to the local time.

receiver, $S N R_{\min }$, and the maximum interference (with respect to the noise floor) tolerance at the victim receiver, $I_{t h}$. Apart from the distance-aware power control methods described earlier, the time varying nature of the traffic demand from the NGSO earth terminal is taken into account. Since in our system model, we are considering the user terminals (not gateways), the traffic demand from a NGSO earth terminal is not expected to be constant all the time [30]. Following the normalized traffic model for the user terminals provided in ITU-R S.1325-3 and [31] (please see Fig. 2), we study the impact of the traffic-aware power allocation on the performance metrics of the FSS system (e.g, interference, SNR, power consumption). Please note that, we are assuming that this traffic model is already known to the network operator (which means the unpredictable traffic patterns are not considered), so as the power allocation based on the provided model can be implemented on the NGSO satellite. As depicted in Fig. 2, the cumulative distribution function (CDF) of the traffic load for a typical NGSO earth terminal varies over time. Therefore, the power of the NGSO satellite per carrier can be varied as a function of the traffic load. We call this technique as a traffic-aware power control (TA-PC) method. We define the three power control methods in the following subsections.

In the rest of this work, the following subscripts are used: $e$ for the earth terminal, $s$ for the satellite, $n$ for the NGSO system, and $g$ for the GSO system.

\subsection{Range-based Power Control Method}

To compute the transmission power on the NGSO satellite as a function of range, $d_{n e, n s}$ (see Fig. 1), we first need to find the SNR level at the NGSO earth terminal. Using the FSL model in ITU-R S.1325-3, the received power at the NGSO earth terminal from the NGSO satellite can be computed as:

$$
\operatorname{Pr} x_{n e}=\operatorname{Pt} x_{n s}\left(d_{n e, n s}\right) G t x_{n s}(0) G r x_{n e}(0) F S L\left(\lambda, d_{n e, n s}\right),
$$

where $\operatorname{Pr} x_{n e}[W]$ is the desired receive power at the input to the NGSO earth terminal antenna, $P t x_{n s}[W]$ is the transmit power of the NGSO satellite station, and function $F S L(\lambda, d)=\left(\frac{\lambda}{4 \pi d}\right)^{2}$, in which $\lambda$ is the wavelength. $G t x_{n s}(0), G r x_{n e}(0)$ are the transmit and receive antenna gains of the NGSO satellite and earth terminal along with the boresight direction, respectively. The received $S N R$ at the NGSO earth terminal is defined as:

$$
S N R=\frac{P t x_{n s}\left(d_{n e, n s}\right) G t x_{n s}(0) G r x_{n e}(0)}{N_{0}}\left(\frac{\lambda}{4 \pi d_{n e, n s}}\right)^{2},
$$

where $N_{0}[W]$ is the thermal noise power at the receiver and can be expressed as:

$$
N_{0}=K \times T_{n e} \times B W_{t x},
$$


where $K$ is the Boltzmann's constant $\left(1.38 \times 10^{-23}[\mathrm{~J} / K]\right)$, and $T_{n e}[K]$ is the noise temperature at the NGSO earth terminal, and $B W_{t x}[H z]$ is the transmit bandwidth. In order to guarantee $S N R_{\text {min }}$ at the NGSO earth terminal the transmit power on the NGSO satellite as a function of range can be computed as follows.

$$
P t x_{n s}\left(d_{n e, n s}\right)=\frac{S N R_{m i n} \times N_{0}}{G r x_{n e}(0) \times G t x_{n s}(0)}\left(\frac{4 \pi d_{n e, n s}}{\lambda}\right)^{2} .
$$

\subsection{Cognitive Range-based Power Control Method}

The range-based power control technique in Eq. (4) satisfies the required $S N R_{\min }$ at the NGSO earth terminal receiver to close the link. To apply the range-based power control in the considered coexistence scenario, we re-write this technique by taking into account the interference threshold level $\left(I_{t h}\right)$ of the GSO earth terminal as following optimization problem.

$$
\begin{array}{ll}
\operatorname{minimize} & \operatorname{Ptx}\left(d_{n e, n s}\right) \\
\text { C.1. } & S N R \geq S N R_{\min } \\
\text { C.2. } & I \leq I_{t h} \\
\text { C.3. } & \text { Ptx }\left(d_{n e, n s}\right) \leq P t x_{\max },
\end{array}
$$

where $P t x_{\max }$ is the available maximum transmission power on NGSO satellite board. In Eq. (5), conditions C.1 is to take care of the NGSO FSS QoS requirement, C.2 is to avoid harmful interference on the GSO earth terminal, and C.3 is to make sure the range-based transmission power allocation does not exceed $P t x_{\max } . I$ is the interference power at the GSO earth terminal, computed by Eq. (6).

$$
I=\frac{\operatorname{Ptx}\left(d_{n e, n s}\right) G t x_{n s}\left(\theta_{n s}\right) G r x_{g e}\left(\theta_{g e}\right)}{F S L\left(\lambda, d_{g e, n s}\right)},
$$

where $\operatorname{Ptx}\left(d_{n e, n s}\right)[W]$ is the available transmit power at the NGSO satellite computed in Eq. (4), $d_{g e, n s}[m]$ is the distance between the GSO earth terminal and NGSO satellite, and $G t x_{n s}\left(\theta_{n s}\right), \operatorname{Gr}_{g e}\left(\theta_{g e}\right)$ are the transmit and receive antenna gains at the off-axis angle of the NGSO satellite and GSO earth terminal respectively. The value of the parameters are given in the Table I.

Depending on the geometry of the earth stations and satellite systems, it is obvious that the optimization problem in Eq. (5) may not have a feasible solution when NGSO satellite is closer to the GSO earth station and far from the NGSO earth station. In this case, although the NGSO satellite can use the maximum power in transmission to close the link with the NGSO earth station receiver, it may not satisfy the interference threshold level at the GSO earth station. This is due to the fact that the threshold constraints in Eq. (5), $S N R_{\min }$, and $I_{t h}$ are a function of $d_{n e, n s}$, and $d_{g e, n s}$ respectively. When the NGSO satellite is getting farther from the NGSO earth terminal, it should increase the transmission power to close the link and provide the $S N R_{\min }$. At the same time, if it is getting closer to the GSO earth terminal, this high transmission power imposes higher $I$ level on the GSO earth terminal. Eventually, it may not satisfy both of the threshold constraints simultaneously. Therefore, it is better to handover the transmission to the next NGSO satellite that has better link conditions.

\subsection{Traffic-aware Power Control Method}

The transmission power on the NGSO satellite, $P t x_{s n}[W]$, can vary based on the traffic model, which means:

$$
P t x_{s n}=C_{\text {traffic }} \times P t_{\text {max }},
$$




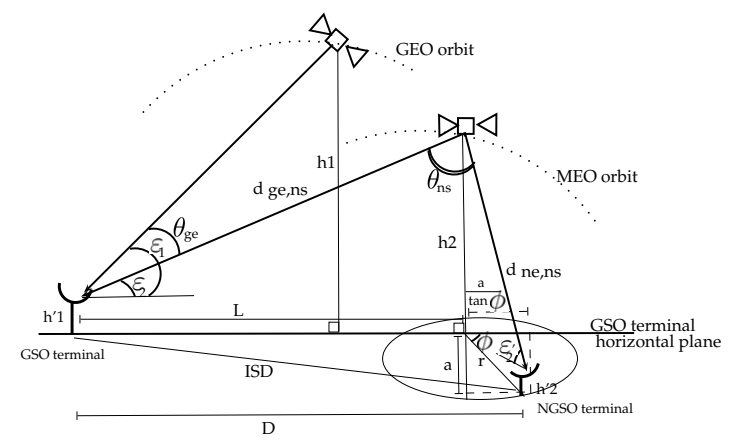

Figure 3. Geometry of GSO and NGSO FSS systems with respect to the ISD.

where $C_{\text {traffic }}$ is the traffic coefficient dependent on the local time, and $P t_{\max }[W]$ is the maximum transmission power of the NGSO satellite. It is assumed that the traffic demand on the NGSO earth terminal regardless of its geometry, varies over time as Fig. 2. By substituting Eq. (7) in Eq. (6), the interference power at the GSO earth terminal can be computed.

In following, we first discuss the geometry determination of ISD between earth stations; then we follow the optimization problem for computing the minimum distance, $I S D_{\text {min }}$, between GSO and NGSO earth terminals.

\section{PROPOSED ISD DETERMINATION METHOD}

In this section, we develop an analytical method for finding the minimum ISD between the GSO and NGSO earth terminals by deploying an optimization problem. Our optimization problem will take into account the interference level from NGSO satellite to the GSO earth terminal in downlink communication. We have assumed that the position of GSO earth terminal is fixed, and we can only control the repositioning of the NGSO earth terminal. Please note that in our ISD model, we have not considered the terrain information such as buildings or mountains. We have assumed that the earth terminals are at the same height above the sea level.

First, let's find a relation between the ISD and the wanted range between NGSO satellite and earth terminal, $d_{n e, n s}$ (please see Fig. (3)). Having the altitude, longitude and latitude of each earth terminal and satellite we can compute distance between any earth terminal to any given satellite $\left(d_{* e, * s}\right)$ as follows [32].

$$
d_{* e, * s}=r_{\text {sat }} \sqrt{1+\left(\frac{R_{E}^{2}}{r_{\text {sat }}}\right)^{2}-2\left(\frac{R_{E}^{2}}{r_{\text {sat }}}\right) \cos \omega \cos \Gamma},
$$

where $r_{\text {sat }}$ is the satellite radius, $R_{E}$ is the earth radius, $\omega$ is the difference in longitude, in degrees, between the earth station and the satellite, and $\Gamma$ is the earth station latitude in degrees. Let's denote local heights of the GSO and NGSO earth terminals as $h_{1}^{\prime}$ and $h_{2}^{\prime}$ respectively. Then, we have:

$$
L=\frac{h_{2}}{\tan \left(\varepsilon_{2}\right)}, h_{2}=d_{n e, n s} \times \sin \left(\varepsilon_{2}^{\prime}\right)+H, H=\left|h_{1}^{\prime}-h_{2}^{\prime}\right|,
$$

where $L$ is the horizontal distance of the NGSO satellite from the GSO earth terminal, $h_{1}$ and $h_{2}$ are the altitudes of the GSO and NGSO satellites from the horizontal plane of the GSO terminal. $\varepsilon_{2}$ is the elevation angle of the NGSO satellite from the GSO earth terminal, and $\varepsilon_{2}^{\prime}$ is the elevation angle of the NGSO satellite from the NGSO earth terminal. From 
Table I. System Parameters

\begin{tabular}{|c|c|}
\hline Parameter & Value \\
\hline Downlink frequency channel & $18.8 \mathrm{GHz}$ \\
\hline$B W_{t x}$ & $125 \mathrm{MHz}$ \\
\hline$G r x_{e n}(\theta), G r x_{e g}(\theta)$ & ITU-R S.1428 \\
\hline$G t x_{s n}(\theta)$ & ITU-R S.1528 \\
\hline$G t x_{s g}(\theta)$ & ITU-R S.672-4 \\
\hline$h_{1}^{\prime}, h_{2}^{\prime}$ & $7 \mathrm{~m}, 12 \mathrm{~m}$ \\
\hline GSO earth antenna diameter & $1.75 \mathrm{~m}[34]$ \\
\hline NGSO earth antenna diameter & $0.75 \mathrm{~m}[33]$ \\
\hline NGSO satellite antenna diameter & $0.361 \mathrm{~m}$ \\
\hline$T_{n e}, T_{g e}$ & $275 \mathrm{~K}$ \\
\hline GSO (Eutelsat) satellite [lat,lon,alt] & {$\left[0^{\circ} S \pm 0.1^{\circ}, 9^{\circ} E \pm 0.1^{\circ}, 35,794 \mathrm{~km}\right]$} \\
\hline NGSO $($ O3b) satellite [lat,lon,alt] & {$\left[1.55^{\circ} S, 31.1^{\circ} E, 8,062 \mathrm{~km}\right]$} \\
\hline GSO terminal [lat,lon] & $\left.7.79^{\circ} S, 24.25^{\circ} E\right]$ \\
\hline $\begin{array}{c}\text { NGSO satellite EIRP in the } \\
\text { direction of the NGSO } \\
\text { receiver earth station }\end{array}$ & $10 \mathrm{dBW}$ \\
\hline$I_{t h}$ & $-10 \mathrm{~dB}$ \\
\hline$S N R_{m i n}$ & $10-15 \mathrm{~dB}$ \\
\hline
\end{tabular}

Eq. (9), we can compute $L$ as:

$$
L=\frac{d_{n e, n s} \times \sin \left(\varepsilon_{2}^{\prime}\right)+H}{\tan \left(\varepsilon_{2}\right)} .
$$

Since $L$ is known, we can compute the horizontal distance between NGSO and GSO earth terminals, $D$, as follows.

$$
\begin{gathered}
r=d_{n e, n s} \times \cos \left(\varepsilon_{2}^{\prime}\right), \\
a=d_{n e, n s} \times \cos \left(\varepsilon_{2}^{\prime}\right) \sin (\phi) ; D=L+\frac{a}{\tan \phi}, \\
\Rightarrow D=\frac{d_{n e, n s}+\sin \left(\varepsilon_{2}^{\prime}\right)+H}{\tan \left(\varepsilon_{2}\right)}+d_{n e, n s} \times \cos (\phi) \cos \left(\varepsilon_{2}^{\prime}\right) .
\end{gathered}
$$

Where $\phi$ is the azimuth angle of the NGSO earth terminal from the GSO earth horizon in degrees. ISD can be computed as

$$
\operatorname{ISD}\left(\varepsilon_{2}, \varepsilon_{2}^{\prime}, \phi, d_{n e, n s}\right)=\sqrt{D^{2}+a^{2}} .
$$

As ISD is a function of $d_{n e, n s}$, we propose an optimization problem for minimizing the ISD as follows.

$$
\underset{d_{n e, n s}}{\operatorname{minimize}} \operatorname{ISD}\left(\varepsilon_{2}, \varepsilon_{2}^{\prime}, \phi, d_{n e, n s}\right)
$$

subject to C.1., C.2., and C.3. in Eq. (5)

By replacing $S N R$ with $S N R_{\min }$ in Eq. (4), and from Eq. (6), the conditions $C .1$ and C.2 in Eq. (13) give us the following restriction on the distance between the NGSO satellite and earth terminal systems.

$$
d_{n e, n s} \leq \sqrt{\frac{I_{t h}}{S N R_{\min }}} \times\left(\frac{F\left(d_{g e, n s}, \theta_{n s}, \theta_{g e}\right)}{C}\right) .
$$

By assuming the off-axis angles, and $d_{g e, n s}$ are not changing, $F$ can be considered as a constant term. The constant term $C$ contains the antenna gain of the NGSO systems on boresight angle $(\theta=0)$. Therefore, by substituting $d_{n e, n s}$ from Eq. (14) into Eq. (12), the minimum value for ISD can be computed.

In next section, we represent the results for the range-based, cognitive and traffic-aware power control mechanisms in the co-existence of GSO-NGSO FSS systems. We also compare the results for $I S D_{\min }$, using the analytical model in Eq. (12), and the simulation results. 


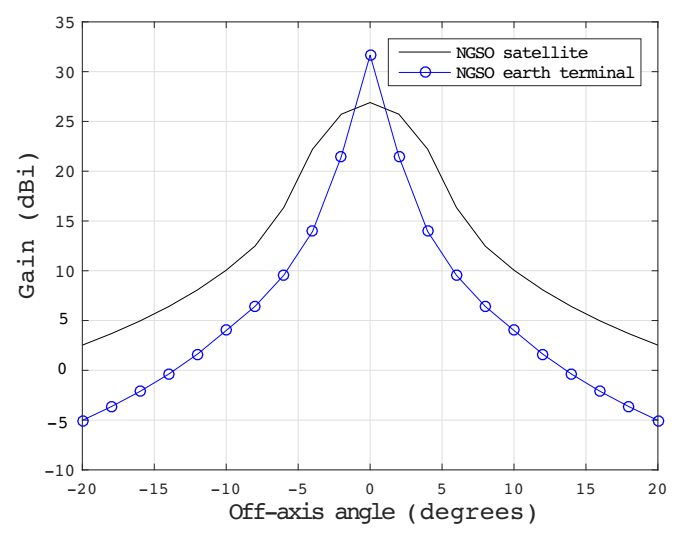

Figure 4. Antenna gain pattern of NGSO FSS system in downlink.

\section{NUMERICAL AND SIMULATION RESULTS}

In this section, first, we show the antenna gain pattern of the NGSO satellite system and earth terminal in Fig. 4. The antenna gain patterns for FSS system in Ka-band are recommended by ITU-R radio regulations as provided in Table I. Then, we compare the results of the $\mathrm{R}-\mathrm{PC}$ with $\mathrm{C}-\mathrm{PC}$ algorithm by assuming there is no available precise traffic demand for NGSO earth terminal. After that, we consider the traffic model given in Fig. 2 for the NGSO earth terminal. We have assumed that the traffic statistics does not vary with geographic location or service type, and it is identical everywhere on the Earth. We show the results for the interference to the noise ratio at the victim receiver by deploying a traffic-aware version of R-PC, and C-PC in comparison with TA-PC. Finally, we explain a Monte Carlo technique, which is conducted for computing the $I S D_{\min }$ in our simulation, and we compare this results with the analytical model provided in Section 4.

Throughout our numerical results, it is assumed that the earth terminal is in association with the constellation, and tracks the corresponding NGSO space station once a communication link is established. We increased the range, $d_{n e, n s}$, by moving the NGSO satellite on its longitude. The range between NGSO satellite and the NGSO earth terminal is in associate with the elevation angle on the NGSO earth terminal, $\varepsilon_{2}^{\prime}$. Once the elevation angle of the NGSO earth terminal is lesser than the minimum elevation angle, the NGSO earth terminal communicate with the next available NGSO satellite with highest elevation angle $\left(90^{\circ}\right)$. The minimum elevation angle is considered to be $5^{\circ}$ for NGSO earth terminal. To compute the off-axis angle with respect to a station, we have assigned a vector in spherical coordinate system from that station to the other stations. For instance, to find $\theta_{n s}$ in Fig. 3 , we connect a vector between the NGSO satellite and its desired earth receiver (in which, off-axis angle $=0^{\circ}$ ), let's call it as V1. Then we connect V2 as the interfering vector between the NGSO satellite to the GSO earth terminal. The angle between vectors, V1, and V2 gives us the observed off-axis angle $\left(\theta_{n s}\right)$ with respect to the NGSO satellite.

\subsection{Results with Uniform Traffic Demand from NGSO Earth Terminal}

In this subsection, we have assumed that the traffic demand from the NGSO earth terminal doesn't vary with the time.

Figure 5 compares the transmission power of the NGSO satellite operating with the R-PC algorithm, which represented in Section 3 in comparison with when the NGSO satellite doesn't utilize any power control mechanism. As it is depicted in Fig. 5, the NGSO satellite without any dynamic power control, operates with the maximum transmission 


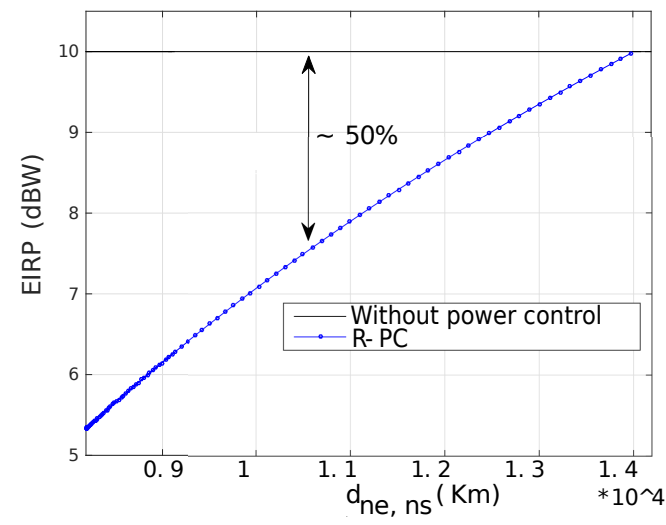

Figure 5. Comparison EIRP for the NGSO satellite system using the range-based power control, $E I R P_{\max }=10 d B W, S N R_{\min }=15 d B$.

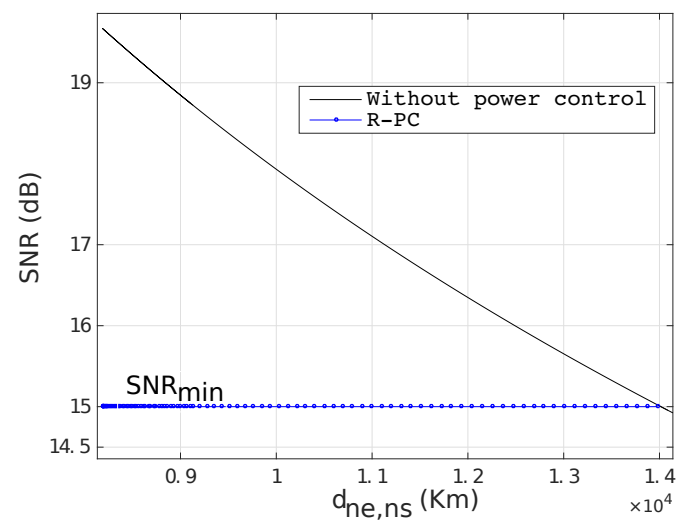

Figure 6. Comparison of received SNR at the NGSO earth terminal using the range-based power control, $P t x_{\max }=10 \mathrm{dBW}, S N R_{\min }=15 \mathrm{~dB}$.

power $\left(E I R P_{\max }=10 \mathrm{dBW}\right)^{\text {ब }}$ constantly. Wherein, with the R-PC, the transmission power of the NGSO satellite gradually increases with respect to the distance range, $d_{n e, n s}$. As it is depicted in Fig. 5, when the distance between the NGSO FSS system is lower than 14000 $\mathrm{km}$, the range-based power control mechanism can approximately save $50 \%$ of the power on-board. The result of SNR level at the NGSO earth terminal using the R-PC algorithm is shown in Fig. 6, where $S N R_{\text {min }}=15 d B$. As it is illustrated in this figure, the received SNR level maintains on the minimum threshold $\left(S N R_{\min }\right)$ with respect to the range. Whereas the received SNR at the NGSO earth terminal is higher than the required $S N R_{\min }$ when the NGSO satellite operates with the maximum power transmission.

In above examples, we didn't implement the C-PC method. To evaluate the effect of the $\mathrm{C}-\mathrm{PC}$ on the interference level of the GSO earth terminal, please see Fig. 7. As it is depicted in the Fig. 7, the $I$ level decreases when the range between NGSO satellite station and GSO earth terminal, $d_{g e, n s}$, increases. However, the R-PC has no limit on the interference level of the GSO, and it can reach above the $I_{t h}$, in smaller $d_{g e, n s}$. Whereas, with the C-PC method, the GSO doesn't receive a harmful in-line interference above the threshold level.

\footnotetext{
"Note that in our numerical calculation we have used the effective isotropically radiated power (EIRP) of the NGSO satellite, which is calculated as $E I R P[d B W]=10 \times\left(\log _{10} \operatorname{Ptx} x_{\max }[W]+\log _{10} G_{t x}(0)\right)$, also we have used equivalent decibel amount of the variables.
} 


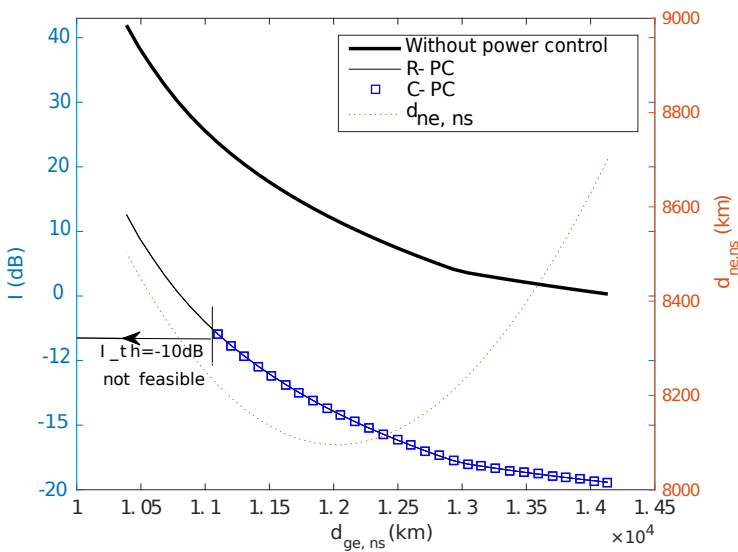

Figure 7. Interference level comparison at GSO earth terminal, using range-based and cognitive power control, $I_{t h}=-10 d B, S N R_{\min }=13 d B, E I R P=10 d B W$.

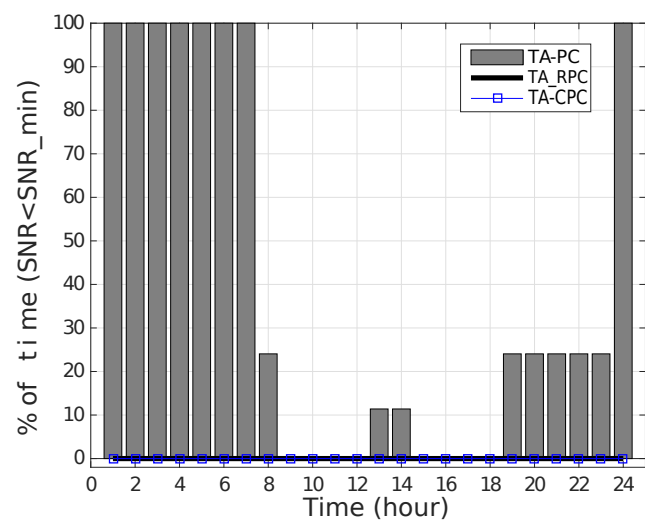

Figure 8. SNR level at NGSO earth terminal. $S N R_{\min }=13 d B, E I R P=5 d B W$.

\subsection{Results with Time-Dependent Traffic Demand from NGSO Earth Terminal}

In this subsection, by considering the traffic model in Fig. 2, we compare the results of the interference level on the GSO earth terminal using R-PC, C-PC \& TA-PC dynamic power control methods on the NGSO satellite system.

To compute a traffic-aware range-based power for the NGSO satellite, we first compute the $\operatorname{Ptx}\left(d_{n e, n s}\right)$ from Eq. 4 to close the link between NGSO FSS system. Then, we can compute the transmit power based on the CDF of the traffic demand corresponding to the time from the following equation:

$$
P t x_{s n}\left(t, d_{n e, n s}\right)=C_{\text {traffic }} \times \operatorname{Ptx}\left(d_{n e, n s}\right),
$$

where $C_{\text {traffic }}$ is the equivalent coefficient of the traffic demand in Fig. 2. For instance, $C_{\text {traffic }}=0.1$, for time interval [1:00 - 7:00] am. We name this method as traffic-aware rangebased power control method (TA-RPC). We followed the same for the C-PC method. Except that the initial transmit power for the NGSO satellite with C-PC method is calculated from Eq. 5. We name this method as traffic-aware cognitive power control (TA-CPC) method. SNR level at the NGSO earth terminal versus time is shown in Fig. 8. Where the percentage of the time in which $S N R$ is below the threshold level is compared with different power control mechanisms. A reasonable check point is to compare the maximum interference level $\left(I \geq I_{t h}\right)$ at the GSO earth terminal. Figure 9 shows the percentage of time that level occurs. 


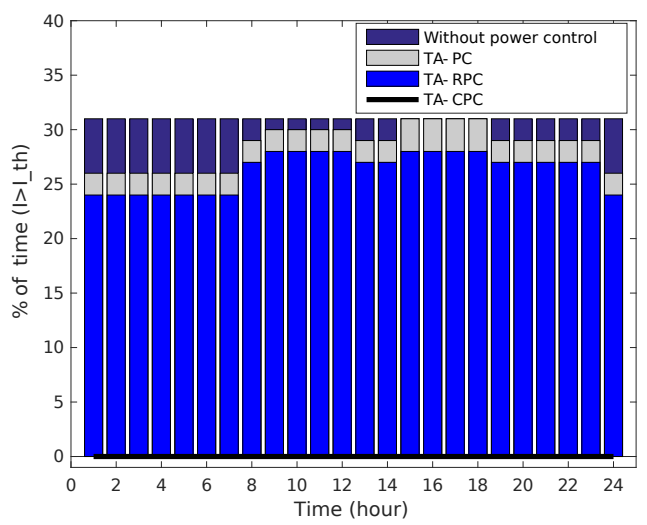

Figure 9. Interference level comparison at GSO earth terminal caused by NGSO satellite system, $I_{t h}=-10 d B, E I R P=5 d B W$.

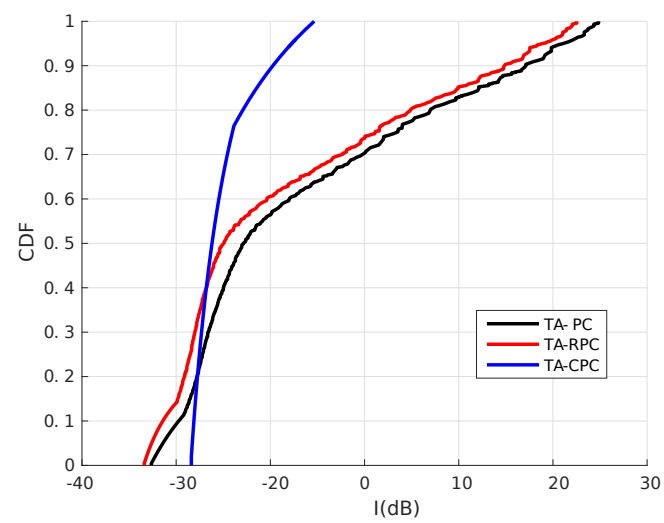

Figure 10. CDF of the interference at GSO earth terminal, using TA-RPC, TA-CPC, \& TA-PC algorithms. $I_{t h}=-10 d B, S N R_{\min }=13 d B, E I R P=5 d B W$.

As it comes from Fig. 9, the proposed TA-CPC method can protect the victim receiver from the harmful in-line interference. We executed the simulation for 24 hours to see a better picture of received $I$ level at the GSO earth terminal. The CDF of the interference at the GSO earth terminal is depicted in Fig. 10, when $I_{t h}=-10 d B$.

\subsection{Monte Carlo method for choosing ISD min between earth terminals and their associated GSO-NGSO satellite coverage}

Herein, we are interested in finding the $I S D_{\min }$ through simulations. To do so, while the positions of the GSO and NGSO satellites are fixed, for N number of times we execute the following steps: 1) a GSO earth terminal location randomly distributed with an associated GSO satellite, and 2) we localize the NGSO earth terminal (which is associated with NGSO satellite) with respect to the azimuth angle of the GSO earth terminal $(\phi)$. In following we describe the simulation we used in each iteration:

1) The GSO earth terminal location is computed by choosing a random latitude from $-45^{\circ}$ to $45^{\circ}$ and a random longitude within $0^{\circ}$ to $45^{\circ}$, which is the coverage of the earth from an O3b satellite, that can be interfered with Eutelsat (GSO) satellite coverage. The longitude of the GSO earth terminal is chosen by a uniform probability distribution within $\left[0^{\circ}-45^{\circ}\right]$. Whereas, to take care of the spherical shape of the earth, the latitude distribution is chosen 


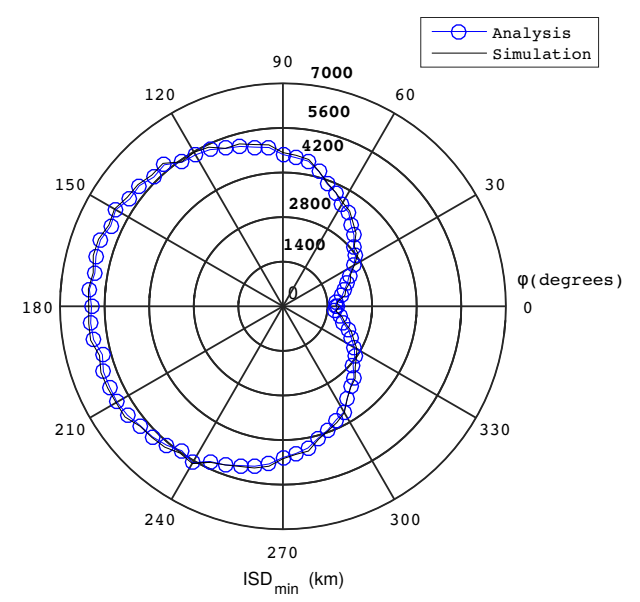

Figure 11. $I S D_{\min }$ between GSO and NGSO earth terminals.

from the following equation.

$$
\text { GSO earth terminal latitude }=F\left(\operatorname{rand}(), 45^{\circ}\right),
$$

where $\operatorname{rand}()$ is a function, which returns a random number between $[0,1]$ uniformly. $F\left(\operatorname{rand}(), 45^{\circ}\right)$ returns a latitude value between $-45^{\circ}$ to $45^{\circ}$, which is defined as follows.

$$
F\left(x, 45^{\circ}\right)=(180 / \pi) \arcsin (\sin (45 \times \pi / 180)(2 x-1)) .
$$

Where $0 \leq x \leq 1$. Once the GSO earth terminal location is chosen, it is tested to see if the elevation angle, $\varepsilon_{1}$, is within the minimum operating elevation angle of the GSO network or not. The minimum elevation angle at the GSO earth terminal location should be greater than or equal to $10^{\circ}$ (ITU-R S.1325 3). If not, this location is not included as one of the locations simulated.

2) Once the GSO earth terminal location is selected, then, with respect to azimuth angle of the GSO terminal, $0^{\circ} \leq \phi \leq 360^{\circ}$, NGSO earth terminal will be localized. For each azimuth angle, to find the $I S D_{\min }$ we increase the distance of the NGSO earth terminal form the GSO earth terminal such that its elevation angle with the associated NGSO satellite is $5^{\circ}$, and the threshold values $S N R_{\min }$ and $I_{t h}$ are satisfied. We have assumed that the computed $I S D_{\min }$ in this worst case scenario, in which $\varepsilon_{2}^{\prime}=5^{\circ}$, is always greater than the minimum required distance between the two earth terminals.

We have compared the result of this simulation with the results of the analysis formula for ISD, using Eq. (12) and (14) in Fig. 11. When the azimuth angle of the NGSO earth terminal with respect to the GSO earth terminal, $\phi$, increases to $180^{\circ}$, the off-axis angle between GSO earth terminal and NGSO satellite gets smaller, therefore the $I S D_{\min }$ increases. This result is compared with when the NGSO satellite operates with the maximum transmission power in Fig. 11. As it comes from this figure, the cognitive range-based power control method can significantly reduce the $I S D_{\min }$ between FSS earth terminals. The relation between $I S D_{m i n}, d_{n e, n s}$ and $\phi$ is shown in Fig. 12.

\section{CONCLUSION AND DISCUSSION}

In this work, we examined three possible dynamic power control approaches and evaluated them as in-line interference mitigation techniques in the co-existence of GSO and NGSO FSS systems. We proposed to employ a cognitive range-based power control mechanism for 


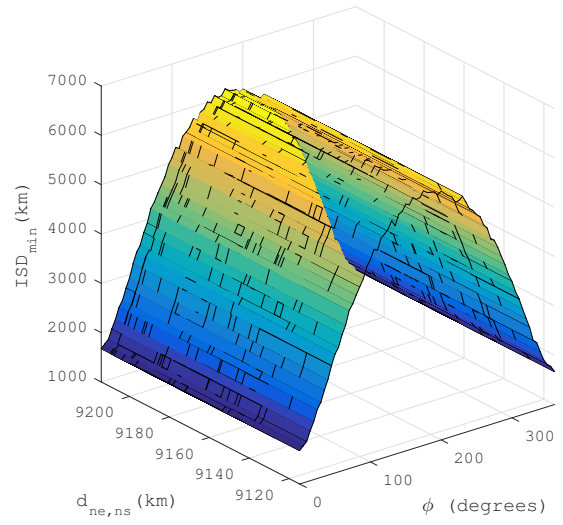

Figure 12. ISD $D_{\min }$ between GSO and NGSO earth terminals with respect to $d_{n e, n s}, \phi$.

an NGSO satellite system operating in the downlink mode. We have compared the proposed power control method with a range-based power control method (recommended by ITU-R S.1325) and a traffic-aware power control mechanism. The results verify that the cognitive power control method avoids the harmful in-line interference at the victim receiver. We also formulated and solved an optimization problem for finding the minimum inter-side distance between the FSS earth stations. We have shown that the numerical results from the proposed minimum inter-site distance model for GSO-NGSO earth terminals verifies the simulation results. The proposed ISD optimization technique can play a critical role in optimizing the network planning strategies. We consider this investigation as our future work.

\section{ACKNOWLEDGEMENT}

This work was supported by the National Research Fund, Luxembourg under the core project HSBNet : Advanced Resource Allocation and Interference Management Strategies for Hybrid Satellite-Terrestrial Backhaul Networks.

\section{REFERENCES}

1. Dae-Sub Oh, Bon-Jun Ku, and Sooyoung Kim. Compatibility study on terrestrial radio system operated in the coverage of multi-beam satellite system. In Communications (APCC), 2011 17th Asia-Pacific Conference on, pages 698-702, Oct 2011.

2. M. Koletta, V. Milas, and Philip Constantinou. Determination of the coordination area for mobile earth stations operating with geostationary space stations in the frequency bands shared with the terrestrial services. In Vehicular Technology Conference, 2005. VTC 2005-Spring. 2005 IEEE 61st, volume 4, pages 2707-2710 Vol. 4, May 2005.

3. Symeon Chatzinotas, Bjorn Ottersten, and Riccardo De Gaudenzi. Cooperative and Cognitive Satellite Systems. Academic Press, 2015.

4. Paul Kolodzy. Spectrum policy task force. Federal Commun. Comm., Washington, DC, Rep. ET Docket, (02-135), 2002.

5. Shree Krishna Sharma, Symeon Chatzinotas, and Björn Ottersten. Satellite cognitive communications: Interference modeling and techniques selection. In Advanced Satellite Multimedia Systems Conference (ASMS) and 12th Signal Processing for Space Communications Workshop (SPSC), 2012 6th, pages 111-118. IEEE, 2012.

6. Symeon Chatzinotas, Sanjay Kumar Sharma, and Bjorn Ottersten. Frequency packing for interference alignment-based cognitive dual satellite systems. In Vehicular Technology Conference (VTC Fall), 2013 IEEE 78th, pages 1-7. IEEE, 2013. 
7. Shree Krishna Sharma, Symeon Chatzinotas, and Björn Ottersten. In-line interference mitigation techniques for spectral coexistence of GEO and NGEO satellites. International Journal of Satellite Communications and Networking, 34(1):11-39, 2016.

8. E. Lagunas, S. Sharma, S. Maleki, S. Chatzinotas, and B. Ottersten. Resource allocation for cognitive satellite communications with incumbent terrestrial networks. Cognitive Communications and Networking, IEEE Transactions on, $\mathrm{PP}(99): 1-1,2015$.

9. Mark J Miller and John C Tchorz. Non-interfering utilization of non-geostationary satellite frequency band for geostationary satellite communication, November 25 2014. US Patent 8,897,769.

10. ECC/DEC/(05)08. The availability of frequency bands for high density applications in the fixedsatellite service (space-to-earth and earth-to-space), 2005

11. Maryan Vazquez-Castro, F Perez-Fontan, and SR Saunders. Shadowing correlation assessment and modeling for satellite diversity in urban environments. International Journal of Satellite Communications, 20(2):151-166, 2002.

12. Rob Rideou. Technologies to identify and/or mitigate harmful interference, url: http://groups.itu. int/br-ssd/Forums/tabid/410/aff/374/aft/803/afv/topic/Default.aspx.

13. Arthur W Wang. Method and apparatus for providing wideband services using medium and low earth orbit satellites, January 13 2004. US Patent 6,678,520.

14. Francesco Vatalaro, Giovanni Emanuele Corazza, Carlo Caini, and Carlo Ferrarelli. Analysis of LEO, MEO, and GEO global mobile satellite systems in the presence of interference and fading. Selected Areas in Communications, IEEE Journal on, 13(2):291-300, 1995.

15. AG Reed and MCJ Posen. Interference in the fixed satellite service bands between the feeder-links of networks using nongeostationary satellites and network using geostationary satellites. In Satellite Communications-ECSC-3, 1993., 3rd European Conference on, pages 251-256. IET, 1993.

16. Sanjay Kumar Sharma, Symeon Chatzinotas, and Bjorn Ottersten. Transmit beamforming for spectral coexistence of satellite and terrestrial networks. In Cognitive Radio Oriented Wireless Networks (CROWNCOM), 2013 8th International Conference on, pages 275-281. IEEE, 2013.

17. Gerard J Foschini and Zoran Miljanic. A simple distributed autonomous power control algorithm and its convergence. Vehicular Technology, IEEE Transactions on, 42(4):641-646, 1993.

18. Roy D Yates. A framework for uplink power control in cellular radio systems. Selected Areas in Communications, IEEE Journal on, 13(7):1341-1347, 1995.

19. Elif Uysal-Biyikoglu, Balaji Prabhakar, and Abbas El Gamal. Energy-efficient packet transmission over a wireless link. IEEE/ACM Transactions on Networking (TON), 10(4):487-499, 2002.

20. Yuhan Zheng, Songlin Sun, Bo Rong, Michel Kadoch, and Yasushi Yamao. Traffic aware power allocation and frequency reuse for green LTE-A heterogeneous networks. In Communications (ICC), 2015 IEEE International Conference on, pages 3167-3172. IEEE, 2015.

21. Alvin C Fu, Eytan Modiano, and John N Tsitsiklis. Optimal energy allocation and admission control for communications satellites. Networking, IEEE/ACM Transactions on, 11(3):488-500, 2003.

22. Francisco Ganhão, Luis Bernardo, Rui Dinis, Gonçalo Barros, Eduardo Santos, António Furtado, Rodolfo Oliveira, and Paulo Pinto. Energy-efficient QOS provisioning in demand assigned satellite NDMA schemes. In Computer Communications and Networks (ICCCN), 2012 21st International Conference on, pages 1-8. IEEE, 2012.

23. Mohammed Hussein, Gentian Jakllari, and Beatrice Paillassa. Network pruning for extending satellite service life in LEO satellite constellations. In Data Science and Data Intensive Systems (DSDIS), 2015 IEEE International Conference on, pages 240-246, Dec 2015.

24. F. Alagoz, O. Korcak, and A. Jamalipour. Exploring the routing strategies in next-generation satellite networks. Wireless Communications, IEEE, 14(3):79-88, June 2007.

25. Wu Di and Li Qing. A new routing algorithm of two-tier LEO/MEO mobile satellite communication systems. In Communications, 2005 Asia-Pacific Conference on, pages 111-115. IEEE, 2005.

26. Charilaos I Kourogiorgas, Pantelis-Daniel Arapoglou, and Athanasios D Panagopoulos. Statistical characterization of adjacent satellite interference for earth stations on mobile platforms operating at ku and ka bands. IEEE Wireless Communications Letters, 4(1):82-85, 2015.

27. Athanasios D Panagopoulos, Theodor D Kritikos, Spiros N Livieratos, and John D Kanellopoulos. Interference studies between adjacent satellite communications systems operating above $10 \mathrm{GHz}$ and using power control as fade mitigation technique. Wireless personal communications, 77(2):1311-1327, 2014.

28. Dae-Sub Oh, Seung-Min Lee, Do-Seob Ahn, and Sooyoung Kim. A study on the separation distance for frequency sharing between GSO network and terrestrial network in ka band. In Vehicular Technology Conference, 2008. VTC Spring 2008. IEEE, pages 2967-2971. IEEE, 2008.

29. IntelSat. A practical introductory guide on using satellite technology for communications. report, http://www.intelsat.com/wp-content/uploads/2013/01/5941-SatellitePrimer-2010.pdf.

30. Btissam Er-Rahmadi, Adlen Ksentini, and Djamal-Eddine Meddour. A traffic-driven analysis for small cells backhaul planning. Small, 2(X2):S1.

31. Jyrki TJ Penttinen. The Telecommunications Handbook: Engineering Guidelines for Fixed, Mobile and Satellite Systems. John Wiley \& Sons, 2015.

32. FestoLab. Satellite orbits, coverage, and antenna alignment. https://www.labvolt.com/downloads/ 87768 f0.pdf.

33. ITU. Working party 4a workshop on earth stations on mobile platforms (esomps). http:// wWw. itu.int/en/ITU-R/study-groups/workshops/RWP4A-2013-ESOMP/Presentations/Addressing\% 20ESOMPs $\% 20$ during $\% 20$ inter-system $\% 20$ coordination $\% 20$ between $\% 20$ satellite $\% 20$ networks.pdf.

34. Eutelsat. Antenna and VSAT type approval / characterization. http://www.eutelsat.com/files/ contributed/satellites/pdf/esog120.pdf. 